Chirurg 2014 · 85:980-992

DOI 10.1007/s00104-014-2809-8

Online publiziert: 12. November 2014

(c) The Author(s). This article is published with open access at link.springer.com
Ulzera, die wegen schlechter Durchblutung des Wundgrunds oder wegen einer Infektion nicht oder verzögert heilen, Infektionen nach Spalthautgittertransplantaten und Defektwunden, bei denen eine plastisch-chirurgische Lappenplastik wegen des Lokalbefunds indiziert, wegen des Allgemeinbefunds aber kontraindiziert ist, gehören zu den alltäglichen Herausforderungen in der septischen und in der plastischen Chirurgie. Randomisierte kontrollierte Studien und Anwendungsbeobachtungen bestätigen, dass Bestrahlungen mit wassergefiltertem Infrarot A (wIRA) helfen, Wunden zu heilen. Wie und warum wIRA die Heilung von Wunden fördert, wird einführend mitgeteilt und an Fallbeispielen die wertvolle Ergänzung chirurgischer Behandlung durch Bestrahlungen mit wIRA veranschaulicht. Der offensichtliche Gewinn für die Behandlung jeder Art von Wunden soll zu weiterer Nutzung von wIRA und zu weiterer Forschung darüber anregen, wie und warum Bestrahlungen mit wIRA Wunden heilen helfen.

\section{Wassergefiltertes Infrarot A}

Wassergefiltertes Infrarot A ist eine spezielle Form der Infrarotstrahlung (Wär-

R. Winkel ${ }^{1} \cdot$ G. Hoffmann ${ }^{2} \cdot$ R. Hoffmann ${ }^{3}$

${ }^{1}$ Chefarzt a.D., Handchirurgie und wiederherstellende Plastische Chirurgie,

Berufsgenossenschaftliche Unfallklinik Frankfurt am Main

${ }^{2}$ Institut für Sportwissenschaften, Johann Wolfgang Goethe-Universität, Frankfurt am Main

${ }^{3}$ Unfallchirurgie und Orthopädische Chirurgie,

Berufsgenossenschaftliche Unfallklinik Frankfurt am Main

\title{
Wassergefiltertes Infrarot A (wIRA) hilft Wunden heilen
}

mestrahlung) im Bereich von 780$1400 \mathrm{~nm}$, die aufgrund ihrer sehr guten Verträglichkeit in der Medizin zu Prävention und Therapie verwendet wird [7].

Wassergefiltertes Infrarot A steigert deutlich

- Temperatur,

- Sauerstoffpartialdruck und

- Durchblutung

im Gewebe $[4,6,7]$. Diese 3 Faktoren sind entscheidend für die ausreichende Versorgung des Gewebes mit Energie und Sauerstoff sowie deshalb auch für die Wundheilung und die Infektionsabwehr $[4,6,7]$.

Wassergefiltertes Infrarot A mindert indikationsübergreifend

- Schmerzen,

- Entzündung und

- vermehrte Sekretion

und verbessert

- Infektionsabwehr und

- Regeneration (bei Wunden sowohl Granulation als auch Epithelialisierung) $[4,7]$.

Wassergefiltertes Infrarot A entspricht dem Großteil der in gemäßigten Klimazonen die Erdoberfläche wassergefiltert erreichenden Infrarotstrahlung der Sonne (Filterwirkung des Wassers und des Wasserdampfs der Erdatmosphäre). Durch die Wasserfilterung werden die Strah- lungsanteile gemindert (die sog. Wasserabsorptionsbanden innerhalb des Infrarot A und die meisten Teile des Infrarot B und $\mathrm{C}$ ), die sonst durch Wechselwirkung mit Wassermolekülen in der Haut eine unerwünschte thermische Belastung (mit stechend-brennendem Gefühl) hervorrufen würden $[4,6,7]$. Technisch wird wIRA in speziellen Strahlern erzeugt (wIRAStrahler), in denen die gesamte Strahlung eines Halogenstrahlers durch eine Wasser enthaltende Küvette hindurchtritt [6, 7]. Während konventionelle Halogenstrahler ohne Wasserfilter („Rotlichtlampen“) je nach korrelierter Farbtemperatur 50$80 \%$ ihrer Strahlung im unerwünschten Infrarot-B- und Infrarot-C-Bereich emittieren, ist dieser Anteil bei wIRA-Strahlern $<0,5 \%$, $\bullet$ Abb. $1[9,17,18]$.

Wassergefiltertes Infrarot A als spezielle Form der Infrarotstrahlung (Wärmestrahlung) mit hohem Eindringvermögen in das Gewebe bei geringer thermischer Oberflächenbelastung erlaubt einen deutlich höheren Energieeintrag in das Gewebe und wirkt sowohl über thermische (auf Wärmeenergietransfer bezogene) und temperaturabhängige (mit Temperaturänderung auftretende) als auch über nichtthermische (ohne relevanten Wärmeenergietransfer) und temperaturunabhängige (ohne relevante Temperaturänderung auftretende) Effekte $[4,6,7]$. 


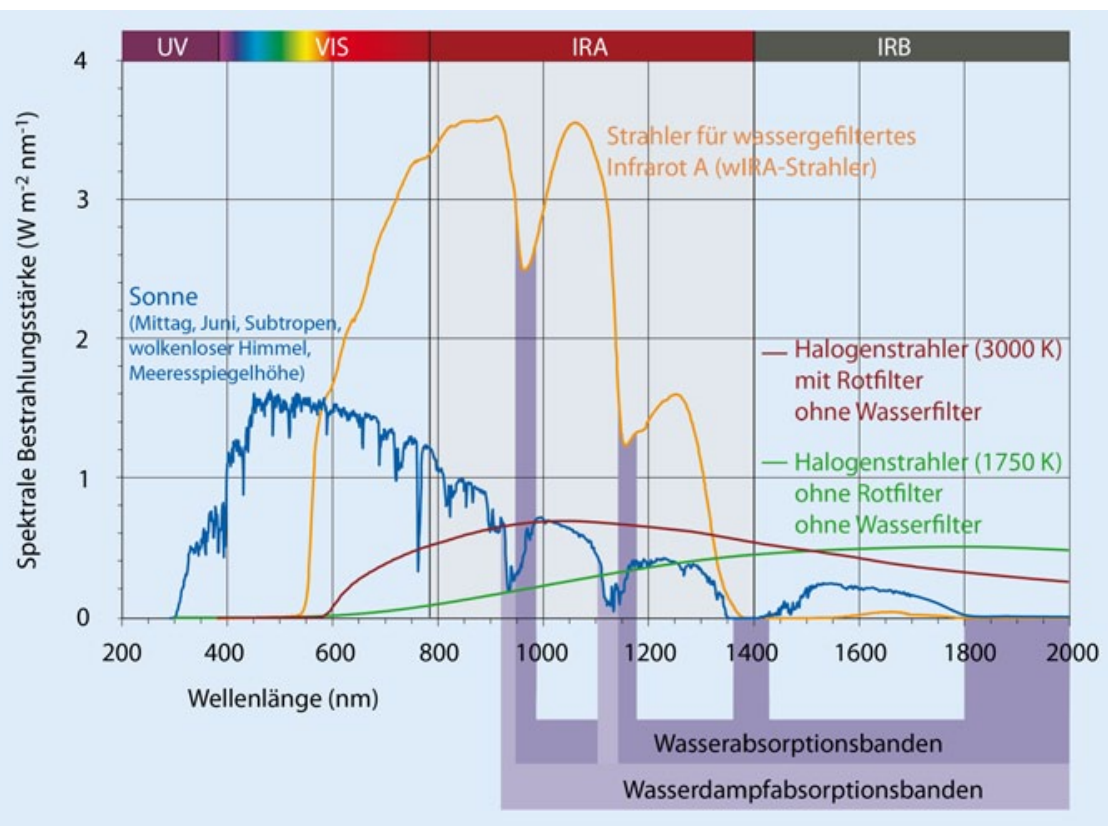

Abb. 1 \ Spektren der Sonne, eines Strahlers für wassergefiltertes Infrarot A und von 2 Halogenstrahlern ohne Wasserfilter: Die 3 verschiedenen Strahler bewirken mit ihren dargestellten spektralen Bestrahlungsstärken die gleiche Hautoberflächentemperatur $[9,17,18]$

Innerhalb des Spektrums der Infrarot-A-Strahlung wurden Effekte von den energiereichen Wellenlängen nahe dem sichtbaren Licht - ungefähr 780-1000 nm, insbesondere $820 \mathrm{~nm}[11]$ - sowohl in vitro als auch in vivo beschrieben. Diese Wellenlängen stellen - v. a. im Hinblick auf nichtthermische Effekte, wie die Minderung der Wundsekretion - den klinisch wichtigsten Teil von Infrarot A und wassergefiltertem Infrarot A dar [7]. Die Bestrahlungsstärken in diesem Wellenlängenbereich sind bei wIRA-Strahlern deutlich höher als bei konventionellen Halogenstrahlern ohne Wasserfilter: Bei einer Wellenlänge von $820 \mathrm{~nm}$ ist die bei gleicher Hautoberflächentemperatur anwendbare Bestrahlungsstärke bei einem wIRA-Strahler beispielsweise ca. 6-mal so hoch wie bei dem in der $\bullet$ Abb. 1 dargestellten Halogenstrahler ohne Wasserfilter mit einer korrelierten Farbtemperatur (,correlated color temperature“, CCT) von $3000 \mathrm{~K}$ und ca. 30-mal so hoch wie bei dem Halogenstrahler ohne Wasserfilter mit einer CCT von $1750 \mathrm{~K}[9,17,18]$.

Außerdem wirkt vermutlich eine Bestrahlung mit sichtbarem Licht (,visible light", VIS) und wIRA in Verbindung mit endogenem Protoporphyrin IX (oder Protoporphyrin IX von Bakterien) gewissermaßen wie eine milde photodynami- sche Therapie (endogener PDT-ähnlicher Effekt) $[6,7,21]$. Dies kann die Zellregeneration und Wundheilung fördern (alte oder vorgeschädigte Zellen gehen in die Apoptose und werden durch neue Zellen ersetzt) und antibakteriell/antiinfektiv wirken (photodynamische Inaktivierung von Bakterien) [7].

Einen wesentlichen Anteil an der antiinfektiven - antibakteriellen und antiviralen - Wirkung von wIRA dürfte die Verbesserung der körpereigenen Abwehr durch Steigerung von Temperatur, Sauerstoffpartialdruck und Durchblutung des Gewebes sowie der damit verbesserten Energie- und Sauerstoffbereitstellung für die körpereigene Abwehr haben, also ein thermischer wIRA-Effekt. Dieser ist kombiniert mit nichtthermischen wIRA-Effekten (z. B. auf immunkompetente Zellen), sodass eine immunmodulatorische Wirkung und eine bessere lokale Immunkompetenz resultieren [7]. Zudem wirkt wIRA auch über die Schmerzminderung antiinfektiv [5], da eine Schmerzminderung - neben einer Verbesserung der Lebensqualität - auch den für die Wundheilung bedeutsamen [16] Sauerstoffpartialdruck erhöht [1] und damit das Infektionsrisiko deutlich senkt [10].

Ausführliche Erörterungen von Wirkmechanismen von wIRA, z. B. über die
Beeinflussung der Cytochrom-c-Oxidase, wurden von Hoffmann $[4,7]$ dargestellt.

\section{Bisherige Studien}

\section{Akute Operationswunden}

\section{Studie Heidelberg}

Eine prospektive, randomisierte, kontrollierte, doppelblinde Studie mit $111 \mathrm{~Pa}$ tienten nach großen abdominalen Operationen in der Klinik für Chirurgie der Universitätsklinik Heidelberg zeigte mit 20 Minuten Bestrahlung 2-mal am Tag (beginnend am 2. postoperativen Tag) in der Gruppe mit wIRA und VIS [wIRA(+VIS), ungefähr 75\% wIRA, 25\% VIS], verglichen mit der Kontrollgruppe mit nur VIS, eine signifikante und relevante Schmerzminderung, verbunden mit einer deutlich verminderten erforderlichen Analgetikadosis: Während 230 einzelner Bestrahlungen mit wIRA(+VIS) nahm der Schmerz der Patienten ausnahmslos ab. Der Median der Schmerzminderung an den postoperativen Tagen 2 bis 6 betrug 13,4 auf einer visuellen 100-mm-Analogskala (VAS 0-100). Der Schmerz in der Kontrollgruppe blieb dagegen unverändert $(\mathrm{p}<0,000001)[2,3$, 6, 7]. Der Median der Schmerzminderung am 3. postoperativen Tag betrug 18,5 gegenüber 0,0, die mediane Differenz zwischen den Gruppen 18,4 (99\%-Konfidenzintervall, $99 \%$-KI, 12,3/21,0, $\mathrm{p}<0,000001$ ) $[2,3,6,7]$.

Die erforderliche Analgetikadosis war in den Untergruppen mit wIRA(+VIS) $52-69 \%$ niedriger (mediane Differenzen) im Vergleich zu den Kontrolluntergruppen mit nur VIS (Median 598 gegenüber $1398 \mathrm{ml}$ Ropivacain, $\mathrm{p}=0,000020$, für Periduralkatheteranalgesie; 31 gegenüber $102 \mathrm{mg}$ Piritramid, $\mathrm{p}=0,00037$, für patientenkontrollierte Analgesie; 3,4 gegenüber $10,2 \mathrm{~g}$ Metamizol, $\mathrm{p}=0,0045$, für i.v.- und orale Analgesie) [2, 3, 6, 7].

Während der Bestrahlung mit wIRA(+VIS) stiegen der subkutane Sauerstoffpartialdruck wesentlich um $32 \%$ und die subkutane Temperatur um $2,7^{\circ} \mathrm{C}$ an (beide in einer Gewebetiefe von $2 \mathrm{~cm}$ ), während beide in der Kontrollgruppe unverändert blieben $[2,3,6,7]$. Nach Bestrahlung betrug der Median des subkutanen Sauerstoffpartialdrucks 41,6 (mit 
wIRA) gegenüber $30,2 \mathrm{mmHg}$ in der Kontrollgruppe [mediane Differenz zwischen den Gruppen 11,9 mmHg (+39\%), 99\%-KI 8,4/15,4 mmHg (+28\%/+51\%), $\mathrm{p}<0,000001]$ und der Median der subkutanen Temperatur 38,9 gegenüber $36,4^{\circ} \mathrm{C}$ (mediane Differenz zwischen den Gruppen $2,6^{\circ} \mathrm{C}, 99 \%$-KI $2,1 / 2,9^{\circ} \mathrm{C}$, $\mathrm{p}<0,000001)[2,3,6,7]$. Die Ruhewerte (vor Bestrahlung) des subkutanen Sauerstoffpartialdrucks stiegen vom 2. zum 10. postoperativen Tag um 3,4 gegenüber $0,3 \mathrm{mmHg}$ [mediane Differenz zwischen den Gruppen 3,1 mmHg (+10\%), 99\%-KI 1,9/3,7 mmHg, $p=0,00051)[2,3,6,7]$. Die Ruhewerte der subkutanen Temperatur stiegen um 0,4 gegenüber $-0,3^{\circ} \mathrm{C}$ (mediane Differenz $0,6^{\circ} \mathrm{C}, 95 \%$-KI $0,2 / 0,8^{\circ} \mathrm{C}$, $\mathrm{p}=0,0074)[2,3,6,7]$. Beides sind Effekte, die über den Zeitraum der einzelnen Bestrahlung hinausreichen $[2,3,6,7]$.

Die Gesamtbeurteilung des Effekts der Bestrahlung einschließlich Wundheilung, Schmerzen und kosmetischem Ergebnis, erhoben mit einer VAS (0-100 mit 50 als Indifferenzpunkt ohne Effekt) durch den Chirurgen (Median 79,0 gegenüber 46,8, mediane Differenz 27,9, 99\%-KI $17,2 / 37,3, \mathrm{p}<0,000001)$ oder den Patienten $(79,0$ gegenüber 50,2 , mediane Differenz 23,8, 99\%-KI 9,5/34,1, p=0,000007), war in der Gruppe mit wIRA wesentlich besser als in der Kontrollgruppe [2, 3, 6, 7]. Das galt auch für die einzelnen Aspekte, d. h. die Wundheilung, erhoben mit einer VAS durch den Chirurgen (Median $88,6$ gegenüber $78,5, \mathrm{p}<0,000001)$ oder den Patienten (Median 85,8 gegenüber 81,0, $\mathrm{p}=0,040$ ), und das kosmetische Ergebnis, erhoben mit einer VAS durch den Chirurgen (Median 84,5 gegenüber 76,5, $\mathrm{p}=0,00027$ ) oder den Patienten (Median $86,7$ gegenüber $73,6, p=0,00077)[2,3,6$, 7].

Außerdem zeigte sich ein Trend zugunsten der wIRA-Gruppe hin zu einer niedrigeren Rate von Wundinfektionen insgesamt (3 von 46, 7\%, gegenüber 7 von $48,15 \%$, Differenz $-8 \%$, 95\%-KI $-20 \% / 4 \%, p=0,21)$, einschließlich später Infektionen nach der Entlassung, hervorgerufen durch eine unterschiedliche Rate später Infektionen nach der Entlassung mit 0 von $46(0 \%)$ in der wIRA-Gruppe und 4 von $48(8 \%)$ in der Kontrollgruppe (Differenz $-8 \%, 95 \%-\mathrm{KI}-18 \% / 2 \%, \mathrm{p}=0,12$ ) [2,

Chirurg 2014 - 85:980-992 DOI 10.1007/s00104-014-2809-8

(c) The Author(s) . This article is published with open access at link.springer.com

\section{R. Winkel · G. Hoffmann · R. Hoffmann \\ Wassergefiltertes Infrarot A (wIRA) hilft Wunden heilen}

\section{Zusammenfassung}

Wassergefiltertes Infrarot A (wIRA) als spezielle Form der Wärmestrahlung mit hohem Eindringvermögen in das Gewebe bei geringer thermischer Oberflächenbelastung fördert die Heilung akuter und chronischer Wunden sowohl über thermische und temperaturabhängige als auch über nichtthermische und temperaturunabhängige Effekte. Wassergefiltertes Infrarot A steigert die Temperatur $\left(+2,7^{\circ} \mathrm{C}\right.$ in einer Gewebetiefe von $\left.2 \mathrm{~cm}\right)$ und den Sauerstoffpartialdruck im Gewebe $(+32 \%$ in einer Gewebetiefe von $2 \mathrm{~cm})$ und die Gewebedurchblutung. Diese 3 Faktoren sind entscheidend für eine ausreichende Versorgung des Gewebes mit Energie und Sauerstoff und deshalb auch für Wundheilung und Infektionsabwehr. Wassergefiltertes Infrarot A hilft sowohl bei der normalen als auch bei der gestörten Wundheilung, indem es Entzündungsreaktionen und erhöhte Wundsekretion mindert, Infektionsabwehr und Regeneration fördert und Wundschmerzen lindern helfen kann. Die genannten Effekte wurden in insgesamt 7 prospektiven Studien (davon 6 randomisierten kontrollierten Studien) belegt, die meisten mit einem Evidenzgrad von la bzw. Ib. Die hier zusätzlich dargestellten Fallbeispiele komplizierter Wundheilungsverläufe illustrieren die belegten Wirkungen von wIRA. Nicht nur in den hier gezeigten 6 Fällen wendeten die Bestrahlungen mit wIRA komplizierte Wundheilungsverläufe zum Besseren und ermöglichten nach ganz unterschiedlich langen Gesamtdauern der Bestrahlungen (in den 6 Fällen: von 51-550 h) und nach verschieden langen Gesamtdauern der Wundpflege, meist nach Transplantation von Spalthautgittern, die Heilung der Wunden. Bei komplizierten Wundheilungsverläufen ersetzt wIRA nicht den Rat und ggf. auch die Behandlung eines erfahrenen plastischen Chirurgen und eines Chirurgen mit der Spezialisierung in septischer Chirurgie. Mit dieser Einschränkung kann wIRA als wertvolle Ergänzung der Behandlung von akuten und chronischen Wunden empfohlen werden.

\section{Schlüsselwörter}

Wundheilung · Regeneration . Infektionsabwehr · Sauerstoffpartialdruck . Evidenzbasierte Medizin

\section{Water-filtered infrared-A (wIRA) promotes wound healing}

\section{Abstract}

Water-filtered infrared-A (wIRA) is a special form of heat radiation with high tissue penetration and low thermal load to the skin surface which promotes the healing of acute and chronic wounds both by thermal and thermic as well as by non-thermal and nonthermic effects. Water-filtered infrared-A increases tissue temperature $\left(+2.7^{\circ} \mathrm{C}\right.$ at a tissue depth of $2 \mathrm{~cm}$ ), tissue oxygen partial pressure $(+32 \%$ at a tissue depth of $2 \mathrm{~cm})$ and tissue perfusion. These three factors are decisive for a sufficient supply of tissue with energy and oxygen and consequently also for wound healing and infection defense. Waterfiltered infrared-A promotes normal as well as disturbed wound healing by diminishing inflammation and exudation, by promotion of infection defense and regeneration, and by alleviation of pain. These effects have been proven in a total of seven prospective studies (of these six randomized controlled studies) with most of the effects having an evidence level of la or lb. The additional cases of com- plicated courses of wound healing presented in this article illustrate the proven effects of WIRA. Not only in the 6 presented cases wlRA turned the complicated courses of wound healing for the better and facilitated the healing of the wounds after varying total times of irradiation (in the 6 cases 51-550 h) and after variable times of wound care and mostly after transplantation of split skin grafts. In complicated courses of wound healing wIRA does not replace consultation and, when indicated, treatment by an experienced plastic surgeon and by a surgeon specialized in septic surgery. With these limitations wIRA can be recommended as a valuable complement for the treatment of acute as well as of chronic wounds.

\section{Keywords}

Wound healing $\cdot$ Regeneration $\cdot$ Infection defense - Oxygen partial pressure - Evidencebased medicine 
$3,6,7]$. Und es gab einen Trend hin zu einem kürzeren postoperativen Krankenhausaufenthalt mit 9 Tagen in der wIRAGruppe gegenüber 11 Tagen in der Kontrollgruppe [mediane Differenz -2 Tage (-18\%), 95\%-KI $-3 / 0$ Tage, $\mathrm{p}=0,022]$ [2, $3,6,7]$.

Das Hauptergebnis der Studie war, dass postoperative Bestrahlung mit wIRA selbst den "normalen" ungestörten Wundheilungsprozess verbessert $[2,3,6$, 7].

\section{Studie München}

Eine weitere prospektive, randomisierte, kontrollierte, doppelblinde Studie der Chirurgischen Klinik der Technischen Universität München mit 400 Patienten, bei der die Patienten vor großen abdominalen Operationen einmalig $20 \mathrm{~min}$ mit wIRA bestrahlt wurden, hat die Ergebnisse der oben dargelegten Studie der Klinik für Chirurgie der Universitätsklinik Heidelberg, bei der die Patienten nach groBen abdominalen Operationen mit wIRA bestrahlt wurden, bestätigt. Dies gilt insbesondere für die deutliche Minderung der gesamten Wundinfektionsrate durch wIRA: 5,1\% (9 von 178) gegenüber $12,1 \%$ (22 von 182) Wundinfektionen (Differenz $-7,0 \%, 95 \%$-KI $-12,8 \% /-1,3 \%, \mathrm{p}=0,017)$. Von den Wundinfektionen insgesamt waren v. a. die späten Infektionen (an den postoperativen Tagen 9 bis 30) unter wIRA deutlich reduziert: 1,7\% (3 von 178) vs. $7,7 \%$ (14 von 182; Differenz -6,0\%, 95\%KI $-10,3 \% /-1,7 \%, \mathrm{p}=0,007$ ) ([12] sowie aus den Daten der Publikation berechnete Werte). Bereits frühere Studien mit technisch anderen Wärmeapplikationen hatten positive Effekte sowohl bei präoperativer [14] als auch bei postoperativer [19] Wärmeanwendung gezeigt.

Anmerkung zu den Studien Heidelberg und München: Die Ergebnisse dieser Studien sprechen für die Kombination einer unmittelbar präoperativen Bestrahlung mit postoperativen Bestrahlungen.

\section{Schwerbrandverletzte Kinder (Studie Kassel)}

Eine prospektive, randomisierte, kontrollierte, doppelblinde Studie mit 45 schwerbrandverletzten Kindern in der Klinik für Kinderchirurgie des Kinderkran- kenhauses Park Schönfeld in Kassel ergab mit täglich 30 Minuten Bestrahlung (ab dem 1. Tag, Tag der Verbrennung als Tag 1) in der Gruppe mit wIRA und VIS [wIRA(+VIS), ungefähr 75\% wIRA, 25\% VIS], verglichen mit der Kontrollgruppe nur mit VIS, eine deutlich schnellere Abnahme der Wundfläche [3, 6, 7]. Am 5. Tag (nach 4 Tagen mit Bestrahlung) wurde entschieden, ob ein chirurgisches Débridement nekrotischen Gewebes wegen tieferer (Grad $2 b$ ) Verbrennungen notwendig war (11 von 21 in der Gruppe mit wIRA, 14 von 24 in der Kontrollgruppe) oder eine konservative Behandlung möglich war (Verbrennungen vom Grad 2a) $[3,6,7]$. Die Patienten mit konservativer Behandlung wurden in der Studie weitergeführt und bis zur vollständigen Epithelialisierung bestrahlt $[3,6,7]$.

Die Patienten in der Gruppe mit wIRA zeigten eine deutlich schnellere Abnahme der Wundfläche: Eine Abnahme der Wundfläche im Median um 50\% wurde bereits nach 7 Tagen, verglichen mit 9 Tagen in der Kontrollgruppe, und eine Abnahme der Wundfläche im Median um $90 \%$ wurde nach 9 Tagen, verglichen mit 13 Tagen in der Kontrollgruppe, erreicht $[3,6,7]$. Nach 9 Tagen betrug der Median der Wundflächenabnahme 89,2\% gegenüber $49,5 \%$, die mediane Differenz zwischen den Gruppen 39,5\% Wundflächenabnahme (99\%-KI 34,4\%/43,0\%, $\mathrm{p}=0,000011)[6,7]$. Die mediane Differenz zwischen den Gruppen bestand bereits nach einem Tag mit $\mathrm{p}=0,00013$ und nach 2, 5, 6, 7, 8, 9, 10 und 11 Tagen mit $\mathrm{p}<0,0001[6,7]$. Außerdem zeigte die Gruppe mit wIRA bessere Ergebnisse hinsichtlich der chirurgischen Gesamteinschätzung der Wunde und hinsichtlich der Einschätzung des Effekts der Bestrahlung, verglichen mit der Kontrollgruppe (Letzteres als Trend bis 3 Monate nach der Verbrennung) $[3,6,7]$.

\section{Experimentelle Wunden (Studie Berlin)}

In einer prospektiven, randomisierten, kontrollierten Studie der Klinik für Dermatologie der Universitätsklinik Charité, Berlin, mit 12 Probanden mit jeweils 4 experimentellen oberflächlichen Wunden (5 mm Durchmesser, Saugblasen- technik) wurde mithilfe der Laser-ScanMikroskopie anhand eines Score-Systems die Stratum-corneum-Bildung (von der ersten Schicht von Korneozyten bis zur vollen Ausbildung) beurteilt. Insbesondere für die Tage 5 bis 7 zeigte sich die schnellste Ausbildung des Stratum corneum bei Wunden, die mit wIRA(+VIS) und Dexpanthenol-Salbe behandelt worden waren. Am zweitschnellsten war wI$\mathrm{RA}(+\mathrm{VIS})$ allein, an 3. Stelle lag Dexpanthenol-Salbe allein, und an letzter Stelle waren die unbehandelten Wunden [3, 6, 7]. Keimzahlbestimmungen der Wunden (alle 2 Tage) machten deutlich, dass wIRA(+VIS) und die Kombination von wIRA(+VIS) mit Dexpanthenol-Salbe in der Lage waren, die Kolonisation mit physiologischer Hautflora bis zum Tag 5 im Vergleich zu den beiden anderen Gruppen (untherapierte Gruppe und Gruppe mit nur Dexpanthenol-Salbe) zu verhindern $[3,6,7]$. Zu allen untersuchten Zeitpunkten war das Maß an Kolonisation unter Therapie mit wIRA(+VIS) allein niedriger (stärker supprimiert) als in der Gruppe mit wIRA(+VIS) und DexpanthenolSalbe $[3,6,7]$.

\section{Akute Wunden, Wundserome und persistierende postoperative Schmerzen}

Während der Rehabilitation nach Hüftund Knieendoprothesenoperationen war durch Bestrahlung mit wIRA(+VIS) die Resorption von Wundseromen und Wundhämatomen sowohl klinisch als auch sonographisch schneller, und die Schmerzen waren reduziert $[3,6,7]$. Auch nach großen offenen urologischen Operationen (Prostatektomien, Nephrektomien) wurde mit wIRA eine schnellere Wundheilung und Wundseromresorption beobachtet $[3,6,7]$. Wassergefiltertes Infrarot A kann erfolgreich bei persistierenden postoperativen Schmerzen z. B. nach Thorakotomie eingesetzt werden $[3,6,7]$. Auch Sonderformen von Wunden, wie Ulzera bei kutaner sklerodermer Graftversus-Host-Erkrankung [13], können mit wIRA erfolgreich therapiert werden. 


\section{Chronische venöse}

Unterschenkelulzera

\section{Studie Basel}

In einer prospektiven, randomisierten, kontrollierten Studie in Basel mit $40 \mathrm{~Pa}$ tienten mit chronischen venösen Unterschenkelulzera (initiale Ulkusfläche $1-12,4 \mathrm{~cm}^{2}$ ) führte eine Bestrahlung mit

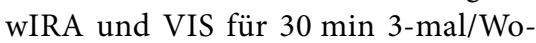
che über einen Zeitraum von 6 Wochen $\mathrm{zu}$ einer signifikant und klinisch relevant schnelleren Wundheilung und einem signifikant und klinisch relevant geringeren Schmerzmittelverbrauch gegenüber einer in gleicher Weise therapierten (Wundsäuberung, antibakterielle Wundauflagen und Kompressionstherapie), aber nicht bestrahlten Kontrollgruppe. Ein vollständiger Wundschluss wurde im Median nach 14 Tagen in der Gruppe mit wIRA gegenüber 42 Tagen in der Kontrollgruppe erreicht (mediane Differenz zwischen den Gruppen -21 Tage; 99\%-KI -28/-7 Tage; $\mathrm{p}=0,000005)$. Nach 42 Tagen waren 19 von 20 Ulzera (95\%) gegenüber 9 von 20 (45\%) Ulzera abgeheilt ( $\mathrm{p}=0,0019)$, und der Median der Restulkusfläche betrug 0 gegenüber $3 \mathrm{~cm}^{2}$ (mediane Differenz zwischen den Gruppen $-3 \mathrm{~cm}^{2} ; 99 \%$ $\left.\mathrm{KI}-4,2 / 0 \mathrm{~cm}^{2} ; \mathrm{p}=0,0091\right)[6,7,8,21]$.

Nach jeder der 6 Wochen war die Zahl der erforderlichen Schmerztabletten pro Tag in der Gruppe mit wIRA signifikant niedriger als in der Kontrollgruppe: Median z. B. nach 2 Wochen 2 Tbl. gegenüber 3 Tbl. (mediane Differenz -1 Tbl.; $\mathrm{p}=0,0029)$, nach 4 Wochen 0 gegenüber 2 Tbl. (mediane Differenz -2 Tbl; $\mathrm{p}=0,000018$ ), nach 6 Wochen 0 gegenüber 2 Tbl. (mediane Differenz -2 Tabletten; 99\%-KI -3/-1 Tbl.; p=0,000005). Der Median der Summe der Tbl. an den 6 Erhebungstagen (der Wochen 1 bis 6) war mit 6 Tbl. in der Gruppe mit wIRA deutlich niedriger als die 14,5 Tbl. in der Kontrollgruppe (mediane Differenz -8 Tbl.; 99\%KI $-12 /-3$ Tabletten; $p=0,000002$ ) [7, 8].

\section{Studie Tromsø/Hillerød}

Eine weitere prospektive Studie der Universität Tromsø, Norwegen, und des Krankenhauses in Hillerød, Dänemark, an 10 Patienten mit aufwendiger thermographischer Verlaufskontrolle ergab unter Therapie mit wIRA(+VIS) eine vollstän- dige oder fast vollständige Abheilung therapierefraktärer chronischer Unterschenkelulzera bei 7 sowie eine deutliche Ulkusverkleinerung bei 2 weiteren der 10 Patienten, eine ausgeprägte Minderung der Schmerzen und des Schmerzmittelverbrauchs (von z. B. 15 auf 0 Schmerztbl. täglich) und eine Normalisierung des thermographischen Bilds (vor Therapiebeginn typischerweise hyperthermer Ulkusrandwall mit relativ hypothermem Ulkusgrund und bis $\mathrm{zu} 4,5^{\circ} \mathrm{C}$ Temperaturdifferenz) $[6,7,15,21]$. Bei einem Patienten wurde ein Ulkus an einem Bein mit dem Vollwirkstrahler [wIRA(+VIS)] therapiert, während ein Ulkus am anderen Bein mit einem Kontrollgruppenstrahler (nur VIS, ohne wIRA) behandelt wurde, was einen deutlichen Unterschied zugunsten der wIRA-Therapie zeigte $[6,7$, $15,21]$.

Alle mit VAS erhobenen Variablen Einschätzung des Effekts der Bestrahlung durch den Patienten und durch den klinischen Untersucher, Einschätzung des Gefühls im Wundbereich durch den Patienten, Einschätzung der Wundheilung durch den klinischen Untersucher sowie Einschätzung des kosmetischen Zustands durch den Patienten und durch den klinischen Untersucher - verbesserten sich während der Bestrahlungstherapieperiode sehr stark; dies entsprach einer verbesserten Lebensqualität $[6,7,15,21]$. Innerhalb der Gruppe der 6 Patienten mit chronischen venösen Unterschenkelulzera ohne Begleitprobleme (d. h. ohne periphere arterielle Verschlusskrankheit, Rauchen oder fehlende venöse Kompressionstherapie) heilten alle 6 Ulzera vollständig oder fast vollständig ab (96-100\% Reduktion der Ulkusfläche) $[6,7,15,21]$.

Die Originalveröffentlichung [15] umfasst auch 10 Anhänge mit detaillierten Informationen zu jedem Patienten und zusätzlich 2 Thermographievideosequenzen.

\section{Studie Freiburg}

In einer prospektiven, randomisierten, kontrollierten, verblindeten Studie der Hautklinik der Universität Freiburg wurden 51 Patienten mit nicht heilenden chronischen venösen Unterschenkelulzera (initiale Ulkusfläche $1-68 \mathrm{~cm}^{2}$ ) mit Kompressionstherapie, Wundsäuberung und nichtadhäsiven Wundauflagen sowie 30 Minuten Bestrahlung 5-mal/Woche über 9 Wochen behandelt $[7,8,20]$. Die Gruppe mit wIRA[+VIS; maximal $140 \mathrm{~mW} / \mathrm{cm}^{2}$ (75\%) wIRA und $45 \mathrm{~mW} /$ $\mathrm{cm}^{2}$ (25\%) VIS] zeigte, verglichen mit der Kontrollgruppe mit nur VIS, eine bessere Gesamteinschätzung der Wundheilung (nach 9 Wochen 85 vs. 67,5 auf einer VAS 0-100, p=0,012), eine höhere Heilungstendenz (nach 9 Wochen bei 84\%, 21 von 25 Patienten, vs. bei $50 \%, 13$ von 26 , $\mathrm{p}=0,023$ ), eine bessere Granulation [nach 9 Wochen bei $84 \%$ ( 21 von 25 ) der Patienten vs. bei $50 \%$ (13 von 26$), \mathrm{p}=0,023$ ] sowie eine Tendenz zu geringerer Exsudation, geringeren Belägen und schnellerer Wundflächenreduktion (Epithelialisierung) [7, 8, 20]. Die Anwendung der wIRA-Strahler bei den Patienten zu Hause erwies sich als sehr gut praktikabel $[7,8,20]$.

\section{Erfahrungen in der Berufsgenossenschaftlichen Unfallklinik Frankfurt am Main}

Die sichtbar positiven Wirkungen von Bestrahlungen mit wIRA auf die Heilung einer Defektwunde nahe dem Sprunggelenk (s. "Fall 1") und Publikationen über die Wirkungen von Bestrahlungen mit wIRA $[2,3,6]$ waren der Anlass zur weiteren Nutzung von wIRA. Wegen des überzeugenden Nutzens für Patienten mit offenen, infizierten und verzögert heilenden Wunden wurden Bestrahlungen mit wIRA seit September 2009 in der Abteilung für Handchirurgie und wiederherstellende Plastische Chirurgie sowie in der Physiotherapie und seit November 2012 auch in der Abteilung für Septische Chirurgie der Berufsgenossenschaftlichen Unfallklinik in Frankfurt a. M. ergänzend in die jeweiligen Behandlungskonzepte bei Wundheilungsstörungen und zunehmend auch bei unkomplizierter Wundheilung eingeführt.

Fallbeispiele zeigen komplizierte Verläufe, bei denen - entgegen der Erwartung der Kliniker - die Bestrahlungen mit wIRA jeweils bei einer überwiegend offenen Wundbehandlung die Heilung entscheidend und richtunggebend förderten.

In den gezeigten Fällen wurden infolge der Vorbereitung durch die Bestrahlungen mit wIRA Heilungen von Wun- 
Tab. 1 Zusammenstellung der Gesamtdauer der Bestrahlungen mit wassergefiltertem Infrarot A (wIRA), der Dauer der Wund pflege sowie der Zeiten zwischen der ersten und der letzten Abbildung pro Fall

\begin{tabular}{|c|c|c|c|}
\hline Fall & $\begin{array}{l}\text { Bestrahlungsdauer, } \\
\text { insgesamt } \\
\text { (h) }\end{array}$ & $\begin{array}{l}\text { Wundpflege bis zur/nach der } \\
\text { Transplantation von Spalthaut } \\
\text { (Tage) }\end{array}$ & $\begin{array}{l}\text { Zeit der Beobachtung } \\
\text { von der ersten bis zur letzten } \\
\text { Abbildung pro Fall (Tage) }\end{array}$ \\
\hline 1 & 550 & 117 , bis & $181(\bullet$ Abb. 2a-f) \\
\hline 2 & 118 & 12 , bis & $108(\bullet$ Abb. 3a,b) \\
\hline 3 & 51 & $103^{a}$, bis & 141 (• Abb. 4a,b) \\
\hline 4 & 74 & $198^{\mathrm{b}}$, bis & $359(\bullet$ Abb. 5a-c) \\
\hline 5 & 252 & 84 , nach & 84 (• Abb. 6a,b) \\
\hline 6 & 60 & Keine zweite Transplantation & $20(\bullet$ Abb. 7a-c) \\
\hline
\end{tabular}

den durch Spalthautgittertransplantate ermöglicht, bei denen eine Lappenplastik indiziert, aber abgelehnt worden (s. Fälle 1, 2 und 3) oder bei denen eine Lappenplastik nicht mehr möglich war (s. Fälle 4 und 5). Im Fall 6 wurde eine zunächst angezeigte 2 . Spalthautgittertransplantation vermieden.

Die Fallbeispiele veranschaulichen:

1. die Verkleinerung von Defekten durch Förderung der Bildung von Granulationsgewebe (Fälle 1 und 2; s. auch die oben genannte Studie Freiburg),

2. die Förderung der Epithelialisierung (Fälle 1, 2 und 5; s. auch die oben genannten Studien Kassel, Berlin, Basel, Tromsø/Hillerød, Freiburg),

3. die Besserung der Durchblutung dystropher Narben (s. Fälle 3 und 4) und

4. die Förderung der Infektionsabwehr (s. Fälle 3, 5 und 6; s. auch die oben genannten Studien Heidelberg, München, Berlin). Die Entzündungszeichen Rötung, Schwellung und Schmerzen bildeten sich unter der Bestrahlung auch in dystropher narbiger Umgebung zurück (s. Fall 3). Stark sezernierende Wunden trockneten (s. Fall 5). Trotz einer Infektion mit multiresistentem Stenotrophomonas maltophilia und mit multiresistentem Staphylococcus epidermidis (s. Fall 1), trotz einer Infektion mit multiresistentem Pseudomonas aeruginosa (s. Fall 5) und auch bei einer Infektion mit Extended-Spectrum- $\beta$ Laktamase (ESBL)-bildenden Klebsiellen (s. Fall 6) heilten Spalthautgittertransplantate.
Die Indikation zu Bestrahlungen mit wIRA wurde gestellt:

1. zur Förderung der Granulationsgewebsbildung bei Defektwunden von Patienten, bei denen eine plastischchirurgische Lappenplastik kontraindiziert war,

2. zur Förderung der Epithelialisierung, spontan und nach Spalthauttransplantaten,

3. zur Besserung der Durchblutung bei Störungen der Wundheilung wegen Minderdurchblutung des Wundgrunds infolge von Narben und/oder von Verschlüssen von Blutgefäßen, die gefäßchirurgisch nicht gebessert werden konnten, und

4. zur Förderung der Infektionsabwehr bei Störungen der Wundheilung infolge Infektion, auch bei Infektionen nach Spalthautgittertransplantaten, und auch

5. zur Förderung der ungestörten Wundheilung.

\section{Durchführung der Bestrahlungen mit wIRA}

Die Dosierung der Bestrahlung wurde individuell angepasst durch Variation der 3 Variablen Anzahl, räumlicher Abstand und Dauer der Bestrahlungen. In der Regel wurde die unbedeckte Wunde 2- bis 3-mal täglich aus einem Abstand von ca. $60 \mathrm{~cm}$ für jeweils $30 \mathrm{~min}$ oder länger bestrahlt, häufig insgesamt $2 \mathrm{~h}$, selten bis zu 5 h/Tag. Der „Wohlfühlabstand“ wurde ermittelt durch schrittweise Annäherung des Strahlers aus einer Entfernung von ca. $100 \mathrm{~cm}$ bis zu einem Abstand, bei dem die Bestrahlung auch bei langen Be- strahlungszeiten als angenehm empfunden wurde.

\section{Offene Wundbehandlung}

Zumindest während der Bestrahlungen wurden die Wunden offen behandelt. Wunden, die nicht sezernierten, wurden mit „Ringer-Lösung“ oder durch Auftragen eines durchsichtigen, klaren Gels ohne Wirkstoff befeuchtet.

Spalthauttransplantate wurden auf feuchtem Wundgrund ab dem 1 . bis 3 . Tag nach der Transplantation bestrahlt.

Nach Lappenplastiken wurde die Indikation zur Bestrahlung schon am 1. Tag nach der Operation gestellt, wenn eine Blässe der Hautinsel oder eine dunklere Färbung des Lappenrands nach Entfernung von Hautfäden zur Verringerung der Spannung der Hautinsel fortbestand.

\section{Dokumentation}

Die Dokumentation der Wunden erfolgte digital fotografisch.

\section{Fallbeispiele}

Die hier dargestellten Fallbeispiele komplizierter Wundheilungsverläufe illustrieren die belegten Wirkungen von wIRA. Nicht nur in den dargestellten 6 Fällen wendeten die Bestrahlungen mit wIRA die komplizierten Wundheilungsverläufe zum Besseren. Die Bestrahlungen mit wIRA ermöglichten nach ganz unterschiedlich langen Gesamtdauern der Bestrahlungen (in den gezeigten Fällen: von 51-550 h) und nach verschieden langen Gesamtdauern der Wundpflege bis zur Transplantation von Spalthautgittern (in den dargestellten Fällen: von 12 bis 198 Tagen) Heilungen von Wunden, bei denen eine Lappenplastik indiziert, aber abgelehnt worden (3 von 6 Fällen) oder bei denen eine Lappenplastik nicht mehr möglich war (2 von 6 Fällen). In einem Fall wurde eine zunächst angezeigte 2. Spalthautgittertransplantation vermieden. Die Zeitspannen von der ersten bis zur letzten Abbildung des jeweiligen Falls betrugen 20 bis 359 Tage ( Tab. 1). 

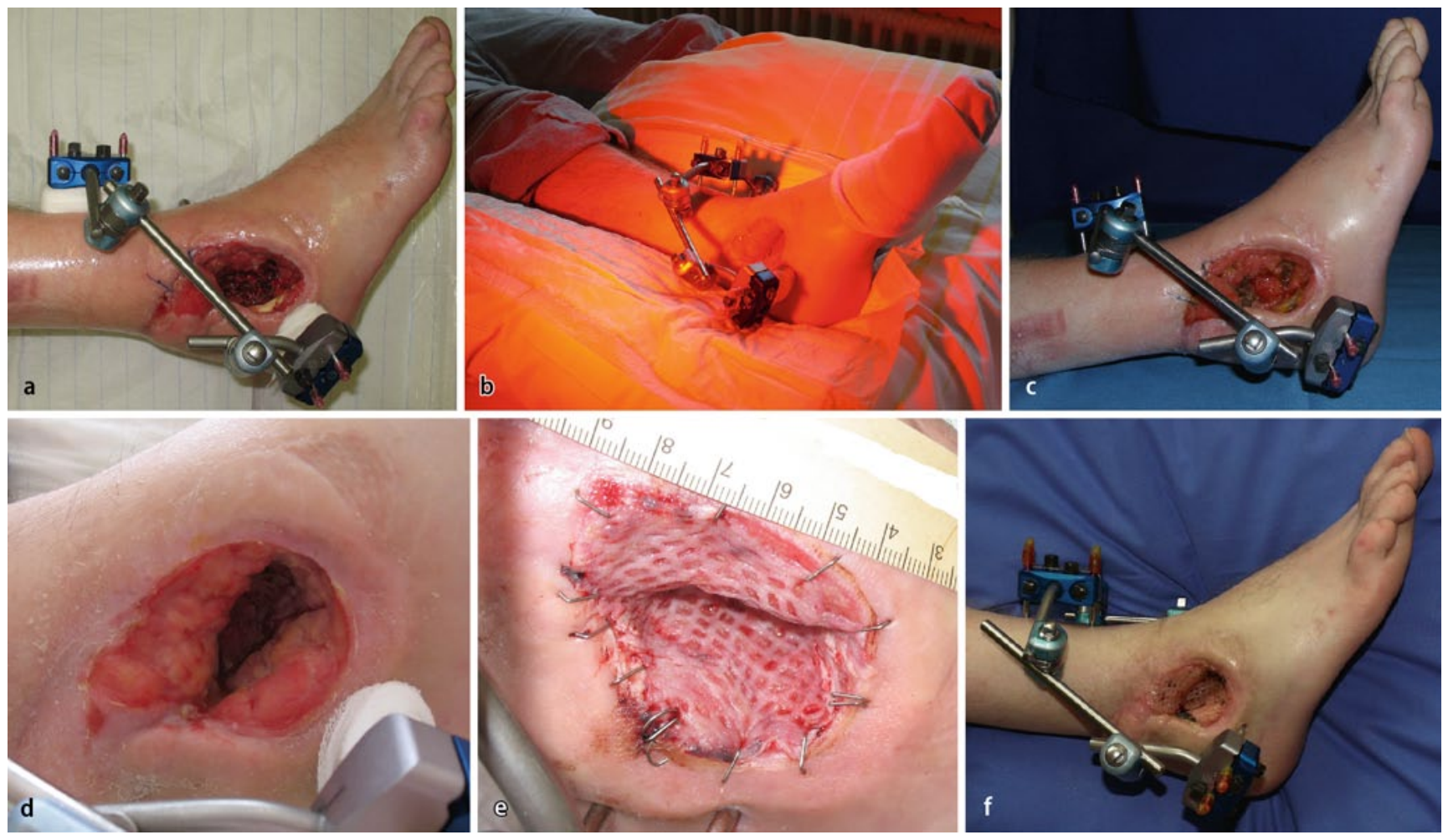

Abb. $2 \Delta$ Fall 1. a Defekt von Weichgewebe und rechtem Außenknöchel nach erneuter Exzision 4 Monate nach Teilnekrose eines Suralislappens. b Bestrahlung mit wassergefiltertem Infrarot A (wIRA). c Unerwartete Granulationsgewebsbildung 10 Tage nach erneutem Beginn der Bestrahlungen, insgesamt $4 \mathrm{~h}$ täglich. $\mathbf{d}$ Während weiterer 90 Tage bestrahlte der Patient bei sich zu Hause, 4-mal $1 \mathrm{~h}$ täglich. Außergewöhnliche Verkleinerung des Defekts durch Granulationsgewebe. Befund 17 Tage vor der erneuten Exzision und der Transplantation eines Spalthautgitters. e Rosiges Spalthautgitter am 2. Tag nach tangentialer Exzision des Granulationsgewebes und Transplantation eines Spalthautgitters. Fortsetzung der Bestrahlungen mit wIRA an 55 Tagen nach der Transplantation des Spalthautgitters täglich etwa 3-mal 30 min. f Fast vollständige Epithelialisierung 64 Tage nach Transplantation des Spalthautgitters und insgesamt 181 Tage nach a

\section{Fall 1}

Zur Zeit der • Abb. 2a 51 Jahre alt, männlich, ohne systemische Erkrankung. Vorgeschichte: 17 Monate vorher Zweietagenfraktur des rechten Femurs und Luxationsfraktur des rechten Sprunggelenks mit Beteiligung der Fußwurzel, Osteosynthesen des Femurs und des Sprunggelenks sowie externe Fixation des Sprunggelenks gelenküberbrückend. Am Sprunggelenk Entfernung des implantierten Metalls 5 Wochen nach der Osteosynthese, gefolgt von mehrfachen Wundexzisionen und Vakuumversiegelungen. Dreizehn Monate nach dem Unfall anderenorts Suralislappen wegen Weichgewebsdefekt. Nekrose der Hauthalbinsel des Lappens, mehrfache Exzisionen und Vakuumverbände. Infektion mit multiresistentem Stenotrophomonas maltophilia und mit multiresistentem Staphylococcus epidermidis.

Die angezeigte und empfohlene erneute Lappenplastik lehnte der Patient ab.
Zehn Tage nach dem letzten radikalen Débridement ( $\bullet$ Abb. 2a) war in der Defektwunde unerwartet viel Granulationsgewebe entstanden, nachdem der Patient die Wunde täglich insgesamt $4 \mathrm{~h}$ (4-mal $1 \mathrm{~h})$ mit wIRA bestrahlt hatte (- Abb. 2b,c). Der zur Zeit der - Abb. 2a zwischen eröffneter Spongiosa der Fußwurzel freiliegende Sprunggelenkspalt und die freiliegende Sehne des M. peronaeus waren nach weiteren 90 Tagen von Granulationsgewebe bedeckt (• Abb. 2d). Das Granulationsgewebe wurde 117 Tage nach dem radikalen Débridement und Bestrahlungen, täglich insgesamt $4 \mathrm{~h}$, tangential exzidiert und ein Spalthautgitter transplantiert (• Abb. 2e; 2 Tage nach der Transplantation, nach einer Bestrahlungspause von 3 Tagen). Die große Defektwunde war 64 Tage nach der Transplantation des Spalthautgitters (Bestrahlungen an 55 Tagen nach der Transplantation des Spalthautgitters täglich etwa 3-mal $30 \mathrm{~min}$ ) und 181 Tage nach dem letzten radikalen Débridement (- Abb. 2a) fast vollständig epithelialisiert (• Abb. 2f). Die indizierte erneute Lappenplastik war nicht mehr dringlich.

Fazit. Der Fall ist Beispiel für die fördernde Wirkung von wIRA auf die Bildung von Granulationsgewebe sowie für die Förderung der Infektionsabwehr und der Epithelialisierung.

\section{Fall 2}

Zur Zeit der • Abb.3a 60 Jahre alt, männlich, massive Polyneuropathie vom Markscheidentyp, Polymyalgia rheumatica, Entfernung beider V. saphenae magnae 1998, postthrombotisches Syndrom nach tiefer linksseitiger Beinvenenthrombose 2004. Infolge der Grunderkrankung Druckulkus an der Rückseite der linken Ferse ( $\bullet$ Abb. 3a), das erst seit etwa 6 Wochen bestand und in den 2 Wochen 

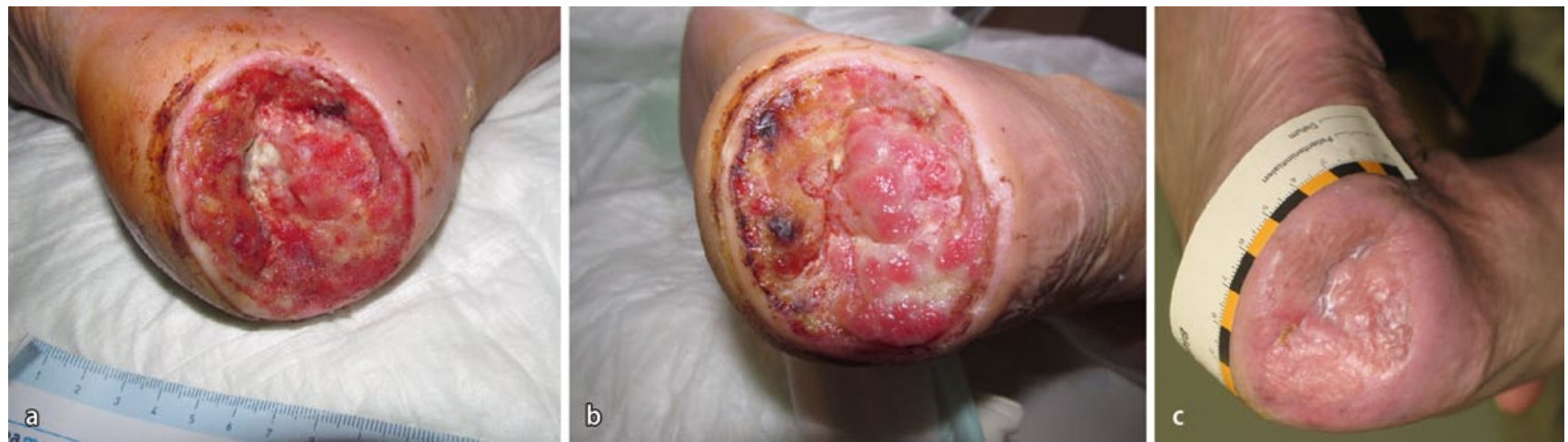

Abb. $3 \Delta$ Fall 2. a Defekt an linker Fersenrückseite, $6 \times 7 \mathrm{~cm}$, vor radikalem Débridement. Indikation zur Lappenplastik, die abgelehnt wurde. b Granulationsgewebsbildung über dem Kalkaneus in nicht erwartetem Ausmaß 6 Tage nach Beginn der Bestrahlungen mit wIRA, 3-mal 30 min täglich. Bei dem Débridement 6 Tage zuvor war die Spongiosa des Kalkaneus tangential eröffnet worden. c Vollständige Epithelialisierung nach 2-maligen tangentialen Exzisionen des Granulationsgewebes, Implantationen des Lederhautersatzes Matriderm ${ }^{\circledast}$ und Transplantationen von Spalthautgittern 108 Tage nach a. Der 2. Eingriff wurde notwendig - nach überwiegender Heilung - wegen Teilverlusts der heilenden Haut infolge unbeabsichtigten Auftretens. Die Bestrahlungsdauer betrug an 49 Tagen täglich 3-mal 30 min bis zur Entlassung, 63 Tage nach a. Zu Hause bestrahlte der Patient weiter täglich 2-mal 30 min bis zur Zeit von c, 108 Tage nach a
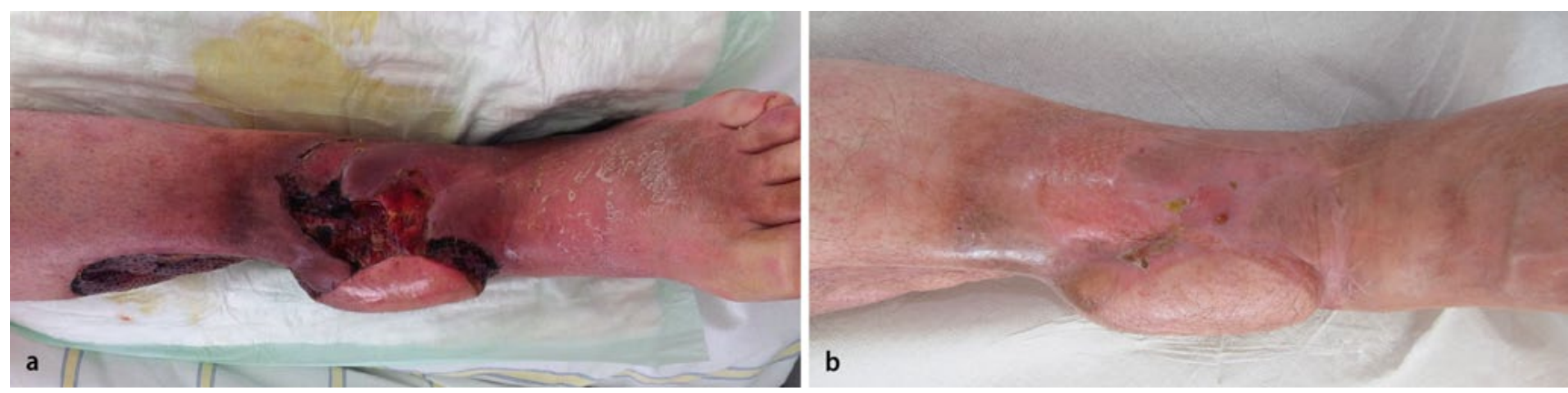

Abb. $4 \Delta$ Fall 3. a Restdefekt in dystropher Umgebung nach Saphenusperforatorlappen. Nach offener Wundbehandlung und Bestrahlungen mit wIRA während der folgenden 5 Tage, 3-mal täglich $1 \mathrm{~h}$, schien wegen des erstaunlich gebesserten Befunds eine Heilung bei konservativer Weiterbehandlung nicht mehr ausgeschlossen. Die Bestrahlungen wurden in gleicher Dosierung bis zur Entlassung fortgesetzt, 14 Tage nach $\mathbf{a}$. $\mathbf{b}$ In örtlicher Betäubung wurde 103 Tage nach dem Befund von a Spalthautgitter mit einem Durchmesser von etwa $3 \mathrm{~cm}$ transplantiert und ab dem 2. postoperativen Tag 6 Tage täglich 3-mal 30 min mit wIRA bestrahlt. Die Wunde war 141 Tage nach dem Befund in a zur Zeit von b ohne weitere Lappenplastik geheilt

zuvor mehrfach exzidiert worden war. Das chronische Ulkus konnte nicht durch einen Suralisperforatorlappen ersetzt werden, weil das Blut aus dem linken Fuß nur durch die V. saphena parva zurückfloss. Mikrobiologischer Nachweis von Pseudomonas aeruginosa und Enterococcus faecalis. Einen indizierten und empfohlenen mikrochirurgisch frei transplantierten Lappen lehnte der Patient ab. Am Tage nach dem radikalem Débridement mit tangentialer Öffnung der Spongiosa des Kalkaneus Beginn der Bestrahlungen mit wIRA, 3-mal täglich 30 min. Nach $6 \mathrm{Ta}$ gen Granulationsgewebsbildung in nicht erwartetem Ausmaß in der Defektwunde, überwiegend über der gut durchbluteten Spongiosa des Kalkaneus (• Abb. 3b). Vollständige Epithelialisierung $108 \mathrm{Ta}$ - ge nach dem radikalen Débridement (• Abb. 3c). Am 13. und am 42. Tag war das Granulationsgewebe tangential exzidiert, zum Ersatz der fehlenden Lederhaut Matriderm $^{\circledR}$ (Kollagen-Elastin-Matrix für die Hautregeneration) implantiert und Spalthautgitter transplantiert worden. Der 2. Eingriff war - nach überwiegender Heilung - notwendig geworden, weil der Patient unbeabsichtigt mit dem Fuß aufgetreten war. Die tiefe Furche zwischen der beweglichen Fußsohlenhaut und dem unbeweglichen Granulationsgewebe über der Spongiosa des Kalkaneus wurde durch die Vaskularisierung und durch die Epithelialisierung des Lederhautersatzes Matriderm ${ }^{\bullet}$ überbrückt (• Abb. 3c). Die zunächst für unerlässlich gehaltene mikrochirurgisch freie Lappenplastik war zumindest nicht mehr dringlich. Die Bestrahlungsdauer betrug bis auf Pausen von jeweils einer Woche nach den Transplantationen 3-mal täglich $30 \mathrm{~min}$ bis zur Entlassung, 63 Tage nach $\bullet$ Abb. 3a. Zu Hause bestrahlte der Patient weiter 2-mal täglich 30 min bis zur Zeit der $\bullet$ Abb. 3c, 108 Tage nach $\bullet$ Abb. 3 a.

Fazit. Auch dieser Fall ist Beispiel für die fördernden Wirkungen von wIRA v. a. auf die Bildung von Granulationsgewebe und auf die Epithelialisierung.

\section{Fall 3}

Zur Zeit der • Abb.4a 54 Jahre alt, männlich, ohne bekannte wesentliche systemische Erkrankung. Offene Fraktur des linken Unterschenkels 11 Jahre zu- 

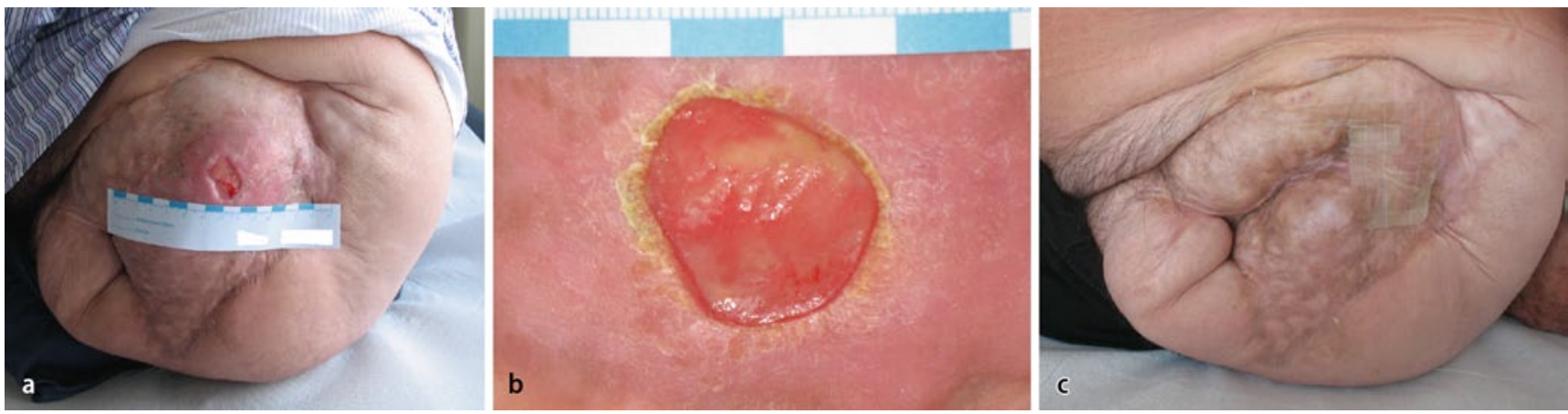

Abb. $5 \Delta$ Fall 4. a Chronisches Druckulkus, $2 \times 3 \mathrm{~cm}$, seit 8 Jahren. Das Ulkus verhinderte die Entfernung des Restes vom Femurhals, der das Druckulkus verursacht hatte und seit 48 Jahren das Gehen mit einer Prothese behinderte. b Bestrahlungen mit wIRA während der vorangegangenen 7 Wochen durch den Patienten bei sich zu Hause, 3-mal täglich 25 min. Subjektiv und objektiv nachhaltige Erwärmung und Rötung der dystrophen Narbe. Änderung der Form, aber keine Verkleinerung des Ulkus, spärliche Bildung von Granulationsgewebe. Fünf Monate nach dem Befund von b wurden 2 Restulzera von je $1 \mathrm{~cm}$ Durchmesser nach präoperativen Bestrahlungen mit wIRA an 10 Tagen, täglich insgesamt 75 min, in örtlicher Betäubung mit 2 Spalthautgittern gedeckt, die heilten. c Bleibend vollständige Epithelialisierung nach Heilung der Spalthautgittertransplantate, ein Jahr nach a und 4 Monate nach erfolgreicher Exartikulation des Femurhalsstumpfes. Die Stücke einer Hydrokolloidfolie dienten dem Hautschutz

vor und seither chronische Osteomyelitis der Tibia im distalen Drittel, 7 Jahre vor der • Abb. 4a mehrfache Revisionen und Einlage einer Septopalkette. Dem Befund in $\bullet$ Abb. 4a waren vorangegangen: 2 Monate zuvor akuter Schub der chronischen Osteomyelitis und Exzisionen, ein Monat zuvor defektangrenzende fasziokutane Lappen in der prätibialen Narbe um den offenen Markraum, Verlust der Lappen wegen fokaler Abszedierungen im Fettgewebe, 2 Wochen zuvor Ersatz des größten Teils des Weichgewebsdefekts über der freiliegenden Tibia durch einen Saphenusperforatorlappen, der heilte. Aber Restdefekt prätibial, mikrobiologischer Nachweis von Proteus vulgaris, Escherichia coli sowie Enterococcus faecalis. In der magnetresonanztomographischen Angiographie: „3-Gefäßversorgung bis zum linken Sprunggelenk“. Einen indizierten und empfohlenen mikrochirurgisch frei transplantierten Lappen lehnte der Patient ab. Der Saphenusperforatorlappen deckte den freiliegenden, eröffneten Teil des Tibiamarkraums, nicht jedoch die proximal davon freiliegende Tibiavorderwand, die mit durchblutetem Periost bedeckt war. Diese Defektwunde war von dystropher Narbe umgeben ( $\mathbf{A b b . 4 a )}$, ein Befund, der kaum Hoffnung auf Heilung ohne einen mikrochirurgisch frei transplantierten Lappen zuließ. Nach offener Wundbehandlung und Bestrahlungen mit wIRA während der folgenden 5 Tage, $3 \mathrm{~h}$ täglich, schien wegen des erstaunlich gebesserten Befunds eine Heilung bei konservativer Weiterbehandlung nicht mehr ausgeschlossen. Die Bestrahlungen wurden in gleicher Dosierung bis zur Entlassung, 14 Tage nach $\bullet$ Abb. 4a, fortgesetzt. Während einer stationären Behandlung wurde 103 Tage nach dem Befund von $\bullet$ Abb. 4a in örtlicher Betäubung ein Spalthautgitter von etwa $3 \mathrm{~cm}$ Durchmesser transplantiert und ab dem 2. postoperativen Tag an 6 Tagen 3-mal täglich 30 min mit wIRA bestrahlt. Die Wunde war 141 Tage nach dem Befund in $\bullet$ Abb. 4a zur Zeit der 1 Abb. $4 b$ ohne weitere Lappenplastik geheilt. Diesen Verlauf hatte der Autor zur Zeit der $\bullet$ Abb. 4a nicht für möglich gehalten.

Fazit. Der Fall ist Beispiel für die fördernden Wirkungen von wIRA v. a. auf die Durchblutung einer dystrophen Narbe, auf die Bildung von Granulationsgewebe, auf die Infektionsabwehr und auf die Epithelialisierung.

\section{Fall 4}

Zur Zeit der - Abb.5a 71 Jahre alt, männlich. Arterielle Hypertonie, Hypercholesterinämie, koronare Herz-Krankheit. Chronisches Ulkus (• Abb. 5a) seit 8 Jahren im Zentrum einer instabilen Narbe über dem Stumpf des linken Femurhalses, nach Amputation vor 48 Jahren, funktionell einer Hüftexartikulation entsprechend. Zusätzlich zum Ver- lust des gesamten linken Beins: rechtsseitiger Fußwurzelstumpf. Seit 4 Jahren Pflasterallergien. Ein Ersatz der instabilen Narbe durch einen großen Bauchhautlappen oder eine mikrochirurgisch frei transplantierte Lappenplastik wäre wegen der Herzerkrankung und der arteriellen Hypertonie mit einem hohen Risiko verbunden gewesen und wurde vom Patienten abgelehnt. Im Abstrich vom Ulkus mikrobiologischer Nachweis von „reichlich Streptococcus agalactiae und reichlich Staphylococcus aureus", sensibel auf die üblichen oralen Antibiotika.

Der Patient bestrahlte das Ulkus 49 Tage bei sich zu Hause 3-mal täglich $25 \mathrm{~min}$ aus $50 \mathrm{~cm}$ Abstand. Er empfand nach eigenem Bekunden 2 Wochen nach dem Beginn der Bestrahlungen die Besserung der Durchblutung als Erwärmung der Narbe. Nach der Vorstellung, bei der der Befund einer gering zunehmenden Granulationsgewebsbildung in dem etwa ebenso großen wie in $\mathbf{A b b}$. 5a, aber runderen Ulkus (• Abb. 5b) fotografiert wurde, und noch im selben Monat wurde der Patient am Herzen operiert (3-facher Koronararterien-Bypass). Rückblickend hatten die Bestrahlungen die Durchblutung der dystrophen Narbe gebessert und die Bildung von spärlichem Granulationsgewebe im Ulkusgrund bewirkt. Offenbar war die Besserung der Durchblutung der dystrophen Narbe so nachhaltig, dass ohne weitere Bestrahlungen knapp 5 Monate nach dem in $\mathbf{A b b} \mathbf{5} \mathbf{b}$ dargestellten 

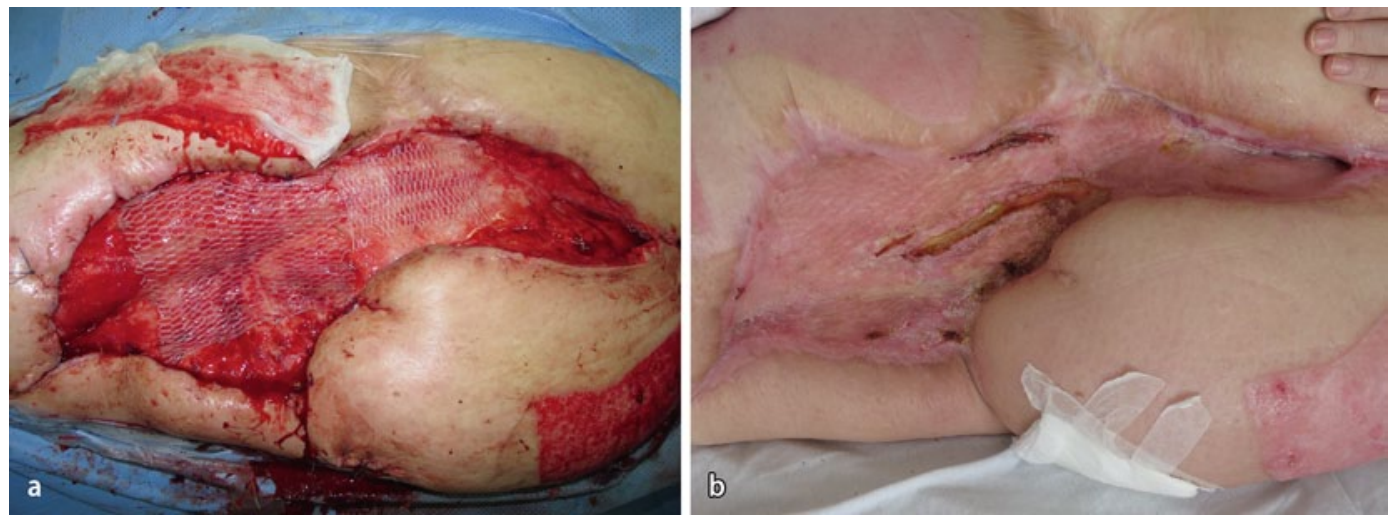

Abb. 6 Fall 5. a Teilweise Deckung eines bis zu $40 \mathrm{~cm}$ langen und bis zu $20 \mathrm{~cm}$ breiten feuchten Weichgewebsdefekts mit Spalthautgitter am Vortag. Infektion mit multiresistentem Pseudomonas aeruginosa. b Epithelialisierung ohne weitere Transplantation von Spalthaut nach 84 Tagen mit 3-mal täglich $1 \mathrm{~h}$ wIRA-Bestrahlung
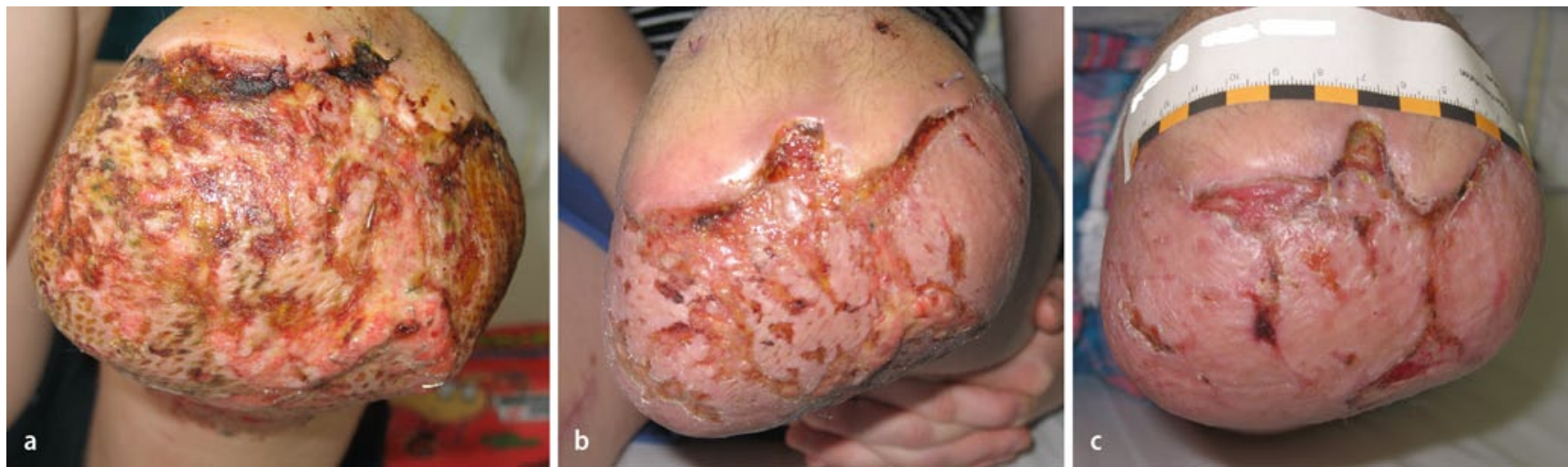

Abb. $7 \Delta$ Fall 6. a Fortschreitende Auflösung transplantierter Spalthautgitter wegen Infektion mit „Extended-spectrum“- $\beta$ Laktamase (ESBL) bildendem Keim, Ansicht der Stumpfkuppe, 9 Tage postoperativ. b Vier Tage nach stationärer Aufnahme und Beginn der Bestrahlungen mit wIRA, 3-mal täglich 1 h. Ansicht der Stumpfkuppe. c Fortschreitende Epithelialisierung 3 Wochen nach Beginn der Bestrahlungen mit wIRA. Ansicht der Stumpfkuppe

Befund nur noch 2 Restulzera von je $1 \mathrm{~cm}$ Durchmesser bestanden. Nach erneuten präoperativen Bestrahlungen über $10 \mathrm{Ta}$ ge, täglich $75 \mathrm{~min}$, verkleinerten sich die Ulzera nicht. Deshalb wurden in örtlicher Betäubung 2 Spalthautgitter transplantiert, die innerhalb einer Woche ohne weitere Bestrahlungen heilten. Die Heilung der chronischen Ulzera ermöglichte die Exartikulation des Femurhalstumpfes. Nach der Exartikulation des Femurhalstumpfes trat kein Druckulkus mehr auf (• Abb. 5c).

Fazit. Der Fall ist ein Beispiel für die fördernde und nachhaltige Wirkung der Bestrahlungen mit wIRA an 59 Tagen auf die Durchblutung einer dystrophen Narbe.

\section{Fall 5}

Zur Zeit der • Abb. 6a 46 Jahre alt, weiblich. Behandlungsrelevante Adipositas. Antikoagulation nach Lungenembolie und Thrombosen, multiple Medikamentenallergien. Luxationsfraktur des linken
Sprunggelenks vor 9 Jahren. Fünf Jahre vor der in $\bullet$ Abb. 6a dargestellten Situation Infektion der Wunde nach Knochenspanentnahme vom linken Beckenkamm zur Arthrodese des linken Sprunggelenks. Seither fortbestehend chronisches Ulkus in linker Leiste und Oberschenkel. Auffallend waren die durch Infektionen komplizierten und tragischen Verläufe nach weit mehr als 100 operativen Eingriffen. Der große in $\bullet$ Abb. 6a sichtbare Weichgewebsdefekt war nach dem Verlust eines großen Schwenk-Verschiebe-Lappens infolge einer Nachblutung bei Gerinnungsstörung und wegen einer Infektion entstanden. Nach der Exzision war der 4 Handflächen große Defekt mit Spalthautgittern gedeckt worden. Diese gingen durch erneute Nachblutung und Infektion bei geschlossener Behandlung vollständig verloren. Zuletzt war der bis zu $40 \mathrm{~cm}$ lange und bis zu $20 \mathrm{~cm}$ breite, feuchte Weichgewebsdefekt nur teilweise mit Spalthautgitter gedeckt worden; die Wunde war mit multiresistentem Pseudomonas aeru- ginosa besiedelt. Die offene Wundbehandlung und die Bestrahlungen mit wIRA an 84 Tagen, 3-mal täglich $1 \mathrm{~h}$, wendeten den jahrelangen Verlauf. Zum Zeitpunkt der Entlassung der Patientin und der Beendigung der Bestrahlungen war die Wunde ohne eine weitere Transplantation von Spalthautgitter fast vollständig epithelialisiert (• Abb. 6b).

Fazit. Der Fall ist ein Beispiel für die Minderung der Sekretion einer großflächigen, mit resistenten Keimen infizierten Defektwunde, für die Förderung der Infektionsabwehr und für die Förderung der Epithelialisierung durch die Bestrahlungen mit wIRA.

\section{Fall 6}

Zur Zeit der • Abb. 7a 15 Jahre alt, weiblich. Infolge einer schwersten Kontusion kam es trotz aller Bemühungen zum Verlust des Unterschenkels. Zum Erhalt der Länge des Femurs wurde im Kniegelenk exartikuliert und mikrochirurgisch ein 
M.-latissimus-dorsi-Lappen mit Monitorinsel über den freiliegenden Stumpf des Femurs transplantiert. Wegen einer Infektion der transplantierten Spalthaut mit ESBL-bildenden Klebsiellen und wegen Phantomschmerz wurde die Patientin 9 Tage nach der Exzision der Monitorinsel und der Spalthautgittertransplantation in die Klinik der Autoren verlegt. Die Exzision der infizierten Spalthaut und eine erneute Spalthautgittertransplantation wurden bei Aufnahme in die Klinik indiziert. Als Alternative wurden Bestrahlungen mit wIRA angeboten. Die Patientin und ihre Eltern wünschten die Bestrahlungen mit wIRA. Bereits 4 Tage nach Beginn der offenen Wundbehandlung mit sehr guter Wundpflege und Bestrahlungen mit wIRA war die fortschreitende Auflösung des Spalthauttransplantats in eine zunehmende Epithelialisierung gewendet ( $\bullet$ Abb. 7b,c). Die bei Aufnahme in die Klinik indizierte Exzision der infizierten Spalthaut und erneute Spalthautgittertransplantation wurden vermieden. Während der Zeit der Beobachtung von - Abb.7a-c wurde an 20 Tagen täglich $3 \mathrm{~h}$ mit wIRA bestrahlt. Die interdisziplinäre Schmerztherapie mit Spiegeltherapie und die Bestrahlungen mit wIRA besserten nach Auskunft der Patientin ihre Schmerzen im Stumpf.

Fazit. Der Fall ist ein Beispiel für die Förderung der Immunabwehr bei einer Infektion mit einem ESBL-bildenden Keim und für die Förderung der Epithelialisierung trotz Infektion.

\section{Evidenz}

Aufgrund von 7 prospektiven klinischen Studien (davon 6 randomisierte kontrollierte Studien) sind die im Folgenden zusammengefassten Wirkungen von wIRA auf die Wundheilung gut belegt (überwiegend mit Evidenzgrad Ia, im Sinne von mehreren prospektiven randomisierten kontrollierten Studien, bzw. Ib), und zwar auf verschiedenartige Wunden (Operationswunden, Verbrennungswunden, chronische Unterschenkelulzera vorwiegend venöser Genese mit und ohne Diabetes mellitus) und anhand verschiedener, sich ergänzender Zielvariablen und Verfahren:
- akute Schmerzminderung während einer wIRA-Bestrahlung (Evidenzgrad Ib): ausnahmslose akute Schmerzminderung bei 230 einzelnen Bestrahlungen, 18,5 vs. 0,0 auf einer VAS-Skala 0-100, p<0,000001 (Studie Heidelberg) [2, 7],

- Minderung der erforderlichen Schmerzmedikation (Evidenzgrad Ia/ Ib): Operationswunden: 52-69\% weniger Analgetikabedarf, $\mathrm{p}=0,000020$ bzw. 0,00037 bzw. 0,0045 (Studie Heidelberg) [2, 7]. Unterschenkelulzera: 6 vs. 14,5 Tbl., $\mathrm{p}=0,000002$ (Studie Basel) [7],

- größere/schnellere Wundflächenreduktion, schnellere Epithelialisierung (Evidenzgrad Ia/Ib): Verbrennungen: 90\% Wundflächenreduktion nach 9 vs. 13 Tagen, $\mathrm{p}=0,000011$ (Studie Kassel) [3, 7]; Unterschenkelulzera: kompletter Verschluss nach 14 vs. 42 Tagen, $\mathrm{p}=0,000005$ (Studie Basel) [7, 21],

- schnellere Granulation (Evidenz$\operatorname{grad} \mathrm{Ib}$ ): 90 vs. 80 auf einer VAS-Skala $0-100, p=0,036$ (Studie Freiburg) $[8,20]$,

- geringere Exsudation (Evidenzgrad Ib, Trend): 30 vs. 55 auf einer VAS-Skala 0-100, $\mathrm{p}=0,075$, Trend (Studie Freiburg) $[8,20]$,

- geringere Wundbeläge (Evidenzgrad Ib, Trend): 20 vs. 40 auf einer VAS-Skala 0-100, $\mathrm{p}=0,070$, Trend (Studie Freiburg) $[8,20]$,

- bessere Gesamteinschätzung der Wundheilung (Evidenzgrad Ia/Ib): Operationswunden: 88,6 vs. 78,5 auf einer VAS-Skala 0-100, p <0,000001 (Studie Heidelberg) [2, 7]. Unterschenkelulzera: 85 vs. 67,5 auf einer VAS-Skala 0-100, p=0,012 (Studie Freiburg) [8, 20],

- bessere Gesamteinschätzung des Effekts der Therapie (Evidenzgrad Ib): 79,0 vs. 46,8 auf einer VAS-Skala 0-100 mit 50 als Neutralpunkt, $\mathrm{p}<0,000001$ (Studie Heidelberg) [2, 7],

- höherer Gewebesauerstoffpartialdruck während wIRA-Bestrahlung (Evidenzgrad Ib): in einer Gewebetiefe von $2 \mathrm{~cm} \mathrm{41,6} \mathrm{vs.} \mathrm{30,2} \mathrm{mmHg}$, $\mathrm{p}<0,000001$ (Studie Heidelberg) [2, 7],
- höhere subkutane Gewebetemperatur während wIRA-Bestrahlung (Evidenzgrad Ib): in einer Gewebetiefe von $2 \mathrm{~cm} 38,9$ vs. $36,4^{\circ} \mathrm{C}, \mathrm{p}<0,000001$ (Studie Heidelberg) [2, 7],

- besserer kosmetischer Aspekt (Evidenzgrad Ib): 84,5 vs. 76,5 auf einer VAS-Skala 0-100, $\mathrm{p}=0,00027$ (Studie Heidelberg) [2, 7],

- niedrigere Wundinfektionsrate (Evidenzgrad Ib): einmalige präoperative Bestrahlung: $5,1 \%$ (9 von 178 ) vs. $12,1 \%$ (22 von 182) Wundinfektionen insgesamt, $\mathrm{p}=0,017$; späte Wundinfektionen (an den postoperativen Tagen 9 bis 30): $1,7 \%$ (3 von 178 ) vs. $7,7 \%$ (14 von 182 ), $\mathrm{p}=0,007$ (Studie München) ([12] sowie aus den Daten der Publikation berechnete Werte). Postoperative Bestrahlung: 7\% (3 von 46 ) vs. $15 \%$ (7 von 48$), p=0,21$, Trend; späte Wundinfektionen $0 \%$ (0 von 46 ) vs. $8 \%$ ( 4 von 48 ), $\mathrm{p}=0,12$, Trend (Studie Heidelberg) [2, 7],

- kürzerer Krankenhausaufenthalt (Evidenzgrad Ib, Trend): 9 vs. 11 Tage, $\mathrm{p}=0,022$, Trend (Studie Heidelberg) $[2,7]$.

Die Erfahrungen in der Berufsgenossenschaftlichen Unfallklinik Frankfurt am Main mit der Förderung der Wundheilung durch Bestrahlungen mit wIRA werden durch die zitierten Studien erklärt. Bestrahlungen mit wIRA waren kein Ersatz für eine angezeigte chirurgische Behandlung. Wenn eine granulierende Wunde sich unter den Bestrahlungen mit wIRA nicht verkleinerte, wurde Spalthaut als Gitter transplantiert. Die Bestrahlungen mit wIRA halfen v. a. durch die Förderung der Durchblutung, der Granulationsgewebsbildung und der Infektionsabwehr Wunden heilen. Meist linderten sie auch den Wundschmerz. Der Nutzen für die Patienten war so überzeugend, dass weitere Studien den wissenschaftlichen Beweis für das Erfahrungswissen über die zitierten Studien hinaus mehren sollten.

Die Dosierung der Bestrahlungen war individuell sehr unterschiedlich. Deshalb wäre wichtig zu wissen, ob sich, individuell unabhängig, Mindestdosen für die unterschiedlichen Zielvariablen einer nachhaltigen Förderung der Durchblu- 
tung, der Granulationsgewebsbildung, der Infektionsabwehr und der Epithelialisierung ermitteln lassen.

$\mathrm{Da}$ ein einzelner wesentlich gestörter Faktor aus der Fülle an wundheilungsbeeinflussenden Faktoren die Wundheilung stören oder unmöglich machen kann, sollte darauf geachtet werden, dass möglichst alle derartigen Faktoren ungestört sind [15]. Die Komplexität der Wundheilung unter individuell unterschiedlichsten Randbedingungen macht es bisher kaum möglich, individuelle Mindestbestrahlungsumfänge (Zeitspannen in Tagen und tägliche Bestrahlungsdauern) für einzelne wIRA-Effekte anzugeben. In der Regel können wIRA-Effekte durch längere Zeitspannen und größere tägliche Bestrahlungsdauern - bei immer moderater Bestrahlungsintensität - gesteigert werden.

Auf die schmerzlindernde Wirkung von Bestrahlungen mit wIRA wurde bei den Fallbeispielen nicht näher eingegangen, weil dies Thema komplex ist und vom Thema der Betrachtung, der Wundheilung, fortführt.

\section{Fazit für die Praxis}

Aus den dargestellten Studien, aus der Evidenz von wIRA-Effekten und aus langjährigen klinischen Erfahrungen der Autoren mit wIRA ergibt sich:

- Wassergefiltertes Infrarot A ist eine nachweislich geeignete Hilfe zur Heilung von Wunden und deshalb eine wertvolle Ergänzung der interdisziplinären chirurgischen Behandlung (unter Einbeziehung der plastischen und der septischen Chirurgie) sowie der Pflege von Wunden. Die Wundheilung wird durch wIRA gefördert, sowohl bei normalem als auch bei gestörtem Verlauf. Wunden heilen schneller oder verkleinern sich, und Transplantationen von Spalthautgittern und Epithelialisierungen werden ermöglicht. Auch in den dargestellten Fallbeispielen heilten unter dem Einfluss von wIRA Defektwunden, wegen deren Lokalbefund Lappenplastiken angezeigt gewesen wären: Nach Vorbereitungszeiten von einigen Wochen führten die Transplantationen von Spalthautgittern zur Heilung der wesentlich kleineren Wunden.

- Wassergefiltertes Infrarot A ist ein kontaktfreies und verbrauchsmaterialfreies Verfahren.

- In der Regel ist die unbedeckte Wunde täglich mindestens insgesamt $60 \mathrm{~min}$ (oder auch deutlich länger, z. B. 2-6 Stunden pro Tag) mit moderater Bestrahlungsstärke (individuell ermittelter "Wohlfühlabstand“) zu bestrahlen. Es sollte bis zum Abschluss der Wundheilung und lieber öfter und länger mit geringerer Bestrahlungsstärke als kürzer mit höherer Bestrahlungsstärke bestrahlt werden.

\section{Hier steht eine Anzeige.}

\section{Springer}




\section{- Strahler für wIRA können auch vom Patienten zu Hause genutzt werden. Die Berufsgenossenschaften über- nahmen meist die Kosten für die leih- weise Bereitstellung eines wIRA- Strahlers zur Förderung der Wundhei- lung.}

Weitere Fachinformationen zu wassergefiltertem Infrarot A (wIRA): http://www.waermetherapie.com/start/fachartikel.html

\section{Korrespondenzadressen}

\section{Dr. R. Winkel}

Chefarzt a.D.

Handchirurgie und wiederherstellende

Plastische Chirurgie, Berufsgenossenschaftliche Unfallklinik Frankfurt am Main,

Friedberger Landstr. 430,

60389 Frankfurt am Main

rwinkel@unitybox.de

\section{Prof. Dr. G. Hoffmann}

Institut für Sportwissenschaften

Johann Wolfgang Goethe-Universität,

Ginnheimer Landstr. 39,

60487 Frankfurt am Main

Hoffmann@em.uni-frankfurt.de

Danksagung. Die Autoren danken ihren ärztlichen Mitarbeitern sowie den Mitarbeitern der Pflege und des Sekretariats für ihre Aufgeschlossenheit und für ihren persönlichen Einsatz für die Patienten. Den $\mathrm{Pa}$ tienten danken die Autoren für ihr Vertrauen und für ihre Mitarbeit bei der offenen Wundbehandlung. Der Dr. med. h.c. Erwin Braun Stiftung danken die Autoren für die kostenfreie Bereitstellung der Strahler.

\section{Einhaltung ethischer Richtlinien}

Interessenkonflikt. G. Hoffmann ist für die Dr. med. h.c. Erwin Braun Stiftung, eine gemeinnützige Schweizer Wissenschaftsstiftung, die sich im Forschungsbereich des wassergefilterten Infrarot A engagiert, tätig. Keiner der Autoren ist oder war bei einer Firma im Themenbereich wassergefiltertes Infrarot A angestellt oder erhält oder erhielt Gelder oder finanzielle Unterstützung einer derartigen Firma. Deshalb erklären die Autoren, dass kein Interessenkonflikt im Sinne der Guidelines of the International Committee of Medical Journal Editors besteht.

\section{Open Access}

This article is distributed under the terms of the Creative Commons Attribution License which permits any use, distribution, and reproduction in any medium, provided the original author(s) and the source are credited.

\section{Literatur}

1. Akca O, Melischek M, Scheck T et al (1999) Postoperative pain and subcutaneous oxygen tension. Lancet 354:41-42

2. Hartel M, Hoffmann G, Wente MN et al (2006) Randomized clinical trial of the influence of local water-filtered infrared $A$ irradiation on wound healing after abdominal surgery. Br J Surg 93:952-960. DOI 10.1002/bjs.5429

3. Hartel M, Illing P, Mercer JB et al (2007) Therapy of acute wounds with water-filtered infrared-A (wIRA) (review). GMS Krankenhaushyg Interdiszip 2:Doc53. http://www.egms.de/pdf/journals/ dgkh/2007-2/dgkh000086.pdf

4. Hoffmann G (2007) Principles and working mechanisms of water-filtered infrared-A (wIRA) in relation to wound healing (review). GMS Krankenhaushyg Interdiszip 2:Doc54. http://www.egms.de/pdf/ journals/dgkh/2007-2/dgkh000087.pdf

5. Hoffmann G (2008) Wassergefiltertes Infrarot A (wIRA) zur Verbesserung der Wundheilung bei akuten und chronischen Wunden. Wundmanagement 2:72-80. http://publikationen.ub.uni-frankfurt.de/volltexte/2008/5429/

6. Hoffmann G (2009) Water-filtered infrared-A (wl$\mathrm{RA}$ ) in acute and chronic wounds (bilingual review). Wassergefiltertes Infrarot A (wIRA) bei akuten und chronischen Wunden (zweisprachige Übersichtsarbeit). GMS Krankenhaushyg Interdiszip 4:Doc12. DOI 10.3205/dgkh000137. http://www.egms.de/pdf/journals/dgkh/2009-4/ dgkh000137.pdf

7. Hoffmann G (2012) Wassergefiltertes Infrarot $A$ in Chirurgie, Dermatologie, Sportmedizin und weiteren Bereichen. In: Krause R, Stange R (Hrsg) Lichttherapie. Springer, Berlin Heidelberg New York Tokio, S 25-54. http://publikationen.ub.uni-frankfurt. de/frontdoor/index/index/docld/24171

8. Hoffmann G (2012) Wassergefiltertes Infrarot A (wIRA) in Chirurgie und Dermatologie. In:Völker S (Hrsg) Siebentes Symposium „Licht und Gesundheit". Eine Sondertagung der Technischen Universität Berlin und der Deutschen Akademie für Photobiologie und Phototechnologie (DAfP), der Deutschen Gesellschaft für Photobiologie (DGP) und der Deutschen Lichttechnischen Gesellschaft (LiTG), 15.-16.03.2012 in Berlin. Universitätsverlag der Technischen Universität Berlin, Universitätsbibliothek, Berlin, S 202-231. http://publikationen. ub.uni-frankfurt.de/frontdoor/index/index/docld/22797

9. Hoffmann G (2013) Wassergefiltertes Infrarot A (wIRA) in der Dermatologie. Haut Dermatol Allergol Kosmetol 24:228-229. http://publikationen. ub.uni-frankfurt.de/frontdoor/index/index/docld $/ 32142$

10. Hopf HW, Hunt TK, West JM et al (1997) Wound tissue oxygen tension predicts the risk of wound infection in surgical patients. Arch Surg 132:9971004

11. Karu TI (2010) Multiple roles of cytochrome c oxidase in mammalian cells under action of red and IR-A radiation (critical review). IUBMB Life 62:607610

12. Künzli BM, Liebl F, Nuhn P et al (2013) Impact of preoperative local water-filtered infrared $A$ irradiation on postoperative wound healing: a randomized patient- and observer-blinded controlled clinical trial. Ann Surg 258:887-894. DOI 10.1097/ SLA.0000000000000235
13. Marks C, Schumann H (2011) Wassergefiltertes Infrarot $A$ in der Wundtherapie bei kutaner sklerodermer Graft-versus-Host Erkrankung. Z Wundheilung 15(Suppl B):83

14. Melling AC, Ali B, Scott EM, Leaper DJ (2001) Effects of preoperative warming on the incidence of wound infection after clean surgery: a randomised controlled trial. Lancet 358:876-880

15. Mercer JB, Nielsen SP, Hoffmann G (2008) Improvement of wound healing by water-filtered infrared-A (wIRA) in patients with chronic venous stasis ulcers of the lower legs including evaluation using infrared thermography. GMS Ger Med Sci 6:Doc11. http://www.egms.de/pdf/gms/2008-6/000056.pdf

16. Niinikoski J, Gottrup F, Hunt TK (1991) The role of oxygen in wound repair. In: Janssen H, Rooman R, Robertson JIS (Hrsg) Wound healing. Wrightson Biomedical Publishing, Petersfield, S 165-174

17. Piazena $H$, Meffert $H$, Uebelhack R et al (2012) Thermische, schädigende und protektive Wirkungen von Infrarot-Hautbestrahlungen. In: Völker S (Hrsg) Siebentes Symposium „Licht und Gesundheit". Eine Sondertagung der Technischen Universität Berlin und der Deutschen Akademie für Photobiologie und Phototechnologie (DAfP), der Deutschen Gesellschaft für Photobiologie (DGP) und der Deutschen Lichttechnischen Gesellschaft (LiTG), 15.-16.03.2012 in Berlin. Universitätsverlag der Technischen Universität Berlin, Universitätsbibliothek, Berlin, S 161-182

18. Piazena H (2013) Vergleich der Spektren eines Strahlers für wassergefiltertes Infrarot A (wIRA, 1) und der Sonne in den Subtropen bei wolkenlosem Himmel am Mittag im Juni in Meeresspiegelhöhe (2) sowie beispielhaft von zwei unterschiedlichen Halogenstrahlern ohne Wasserfilterung für therapeutische und Wellnessanwendungen (3 und 4). http://commons.wikimedia.org/wiki/File:WIRA-Wiki-GH-017C-de-Spektren-wIRA-Sonne-Halogenstrahler.png

19. Plattner $O, A k c a O$, Herbst F et al (2000) The influence of 2 surgical bandage systems on wound tissue oxygen tension. Arch Surg 135:818-822. DOI 10.1001/archsurg.135.7.818

20. Schumann H, Calow T, Weckesser S et al (2011) Water-filtered infrared $A$ for the treatment of chronic venous stasis ulcers of the lower legs at home: a randomized controlled blinded study. Br J Dermatol 165:541-551

21. Felbert $\mathrm{V}$ von, Schumann H, Mercer JB et al (2007) Therapy of chronic wounds with water-filtered infrared-A (wIRA) (review). GMS Krankenhaushyg Interdiszip 2:Doc52. http://www.egms.de/pdf/journals/dgkh/2008-2/dgkh000085.pdf

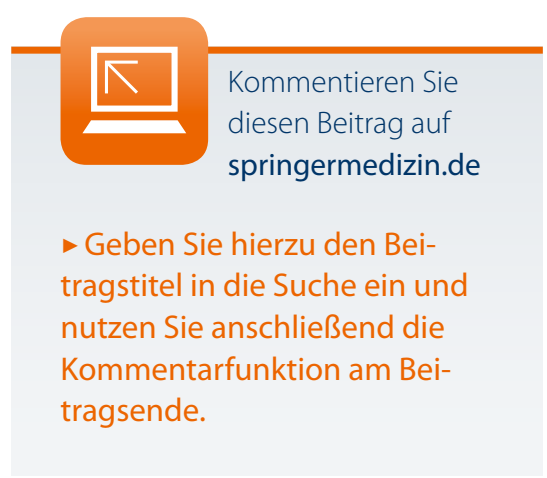

